UDC: $515.146 .2: 515.142 .32$

45 (LXXI) No.2 (2021), 107-112

DOI: $10.37560 /$ matbil $21452107 \mathrm{r}$

\title{
HOMOTOPY OF SYMMETRIC PRODUCTS OF SPHERES
}

\author{
PRAVANJAN KUMAR RANA ${ }^{1}$, BHASKAR MANDAL ${ }^{1}$, AND SARMAD HOSSAIN ${ }^{1,2}$
}

\begin{abstract}
We compute the homotopy groups of infinite symmetric products of spheres. In this paper we show that i) $\pi_{k}\left(S P^{\infty}\left(S^{n}\right)\right)=\left\{\begin{array}{ccc}\mathbb{Z} & \text { for } & k=n \\ 0 & \text { for } & k \neq n\end{array}\right.$, ,

Also, if $m$ and $n$ are distinct nonnegative integers, then using homotopy of infinite symmetric products of spheres we deduce the following results:

ii) $\pi_{k}\left(S P^{\infty}\left(S^{m}\right)\right) \supsetneqq \pi_{k}\left(S P^{\infty}\left(S^{n}\right)\right)$;

iii) $\pi_{k}\left(S P^{\infty}\left(S^{m}\right)\right) \nsim \pi_{k}\left(S P^{\infty}\left(S^{n}\right)\right)$.
\end{abstract}

\section{Introduction}

Now we recall the following definitions and statements.

Algebraic Property of $S^{1}$. Complex numbers can be identified with points in the Euclidean plane $\mathbb{R}^{2}$. Thus the complex number $x+i y$ is identified with the point $(x, y)$ of $\mathbb{R}^{2}$. Under this identification we have the following

i) $S^{1}$ is a group under usual multiplication, it is called circle group and

ii) The circle group $S^{1}$ and the quotient group $\mathbb{R} / \mathbb{Z}$ are isomorphic.

To discuss the higher dimensional spheres $\left(S^{n}(n \geq 1)\right)$ and infinite dimensional sphere $\left(S^{\infty}\right)$, first we discuss the following:

Definition 1.1. Let $X$ be a topological spaces with base point $x_{0} \in X$. For $n \geq 0$, we define the $n$ fold symmetric product of $X$, denoted by $S P^{n} X$ by $S P^{0} X=$ $x_{0}, S P^{n} X=X^{n} / S_{n}$ for $n \geq 1$, where $X^{n}$ denotes the $n$ fold cartesian product of $X$ with itself and $S_{n}$ denotes the symmetric group on $n$ objects regarding as acting on $X^{n}$ by permuting the coordinates. Hence, for $n \geq 1$,

$$
\left.S P^{n} X=\left\{\left(x_{1}, \cdots, x_{n}\right): x_{i} \in X\right)\right\} .
$$

We define $\lim _{n \rightarrow \infty} S P^{n} X=\cup_{n=1}^{\infty} S P^{n} X$ is called an infinite symmetric product of $X$ and is denoted by $S P^{\infty} X$.

2010 Mathematics Subject Classification. 55P65, 55Q40.

Key words and phrases. Homotopy, Infinite symmetric Product, CW-complex, EilenbergMacLane Space. 
P. K. Rana, [10, showed that $S P^{n}$ and $S P^{\infty}$ are covariant functors from the category of pointed topological spaces and base point preserving continuous maps to the category of pointed topological spaces and base point preserving continuous maps.

Definition 1.2. A pointed CW-complex is called an Eilenberg MacLane space if it has only one nontrivial homotopy group. If $G$ is a group and $n$ is a positive integer, the Eilenberg-MacLane space of type $(G, n)$ is a pointed CW-complex $X$ whose homotopy groups vanish in all dimensions except $n$, where $G=\pi_{n}(X)$ and $G$ is to be Abelian for $n>1$, then we can write the notation $K(G, n)$ for a CWcomplex which represents an Eilenberg-MacLane space of type $(G, n)$.

Definition 1.3. Let $X$ be a space and $x_{0}$ a base point of $X$. For a given positive integer $n$, consider the set $F_{n}\left(X, x_{0}\right)$ of all continuous maps $\alpha$ from the unit $n$-cube $I^{n}$ into $X$ for which $\alpha\left(\partial I^{n}\right)=x_{0}$.

Define an equivalence relation $\sim x_{0}$ on $F_{n}\left(X, x_{0}\right)$ as follows:

For $\alpha$ and $\beta$ in $F_{n}\left(X, x_{0}\right), \alpha$ is equivalent module $x_{0}$ to $\beta$ written as $\alpha \sim x_{0} \beta$, if there is a homotopy $H: I^{n} \times I \rightarrow X$ such that:

$$
\begin{aligned}
& H\left(t_{1}, t_{2}, \cdots, t_{n}, 0\right)=\alpha\left(t_{1}, t_{2}, \cdots, t_{n}\right), \\
& H\left(t_{1}, t_{2}, \cdots, t_{n}, 1\right)=\beta\left(t_{1}, t_{2}, \cdots, t_{n}\right),\left(t_{1}, t_{2}, \cdots, t_{n}\right) \in I^{n} \text { and } \\
& H\left(t_{1}, t_{2}, \cdots, t_{n}, s\right)=x_{0},\left(t_{1}, t_{2}, \cdots, t_{n}\right) \in \partial I^{n}, s \in I .
\end{aligned}
$$

Under this equivalence relation on $F_{n}\left(X, x_{0}\right)$, the equivalence class determined by $\alpha$ is denoted by $[\alpha]$ and the homotopy class of $\alpha$ module $x_{0}$ or simply the homotopy class of $\alpha$.

$$
\text { Define }(\alpha \circ \beta)(t)=\left\{\begin{array}{rll}
\alpha\left(2 t_{1}, t_{2}, \cdots, t_{n}\right) & \text { if } \quad 0 \leq t_{1} \leq \frac{1}{2}, \\
\beta\left(2 t_{1}-1, t_{2}, \cdots, t_{n}\right) & \text { if } \quad \frac{1}{2} \leq t_{1} \leq 1 .
\end{array}\right.
$$

With this operation, the set of equivalence classes of $F_{n}\left(X, x_{0}\right)$ is a group called the $n$-th homotopy group of $X$ at $x_{0}$ denoted by $\pi_{n}\left(X, x_{0}\right)$.

Definition 1.4. For every pointed space $\left(X, x_{0}\right)$ and every integer $n \geq 0$, the $n$-th homotopy set as by the $\pi_{n}\left(X, x_{0}\right)=\left[\left(S^{n}, s_{n}\right) ;\left(X, x_{0}\right)\right]$. For $n \geq 2, \pi_{n}\left(X, x_{0}\right)$, is called higher order homotopy group and is sometimes called an absolute homotopy group.

The higher homotopy group has some interesting properties different from fundamental group. If $n>1$, there exists a rotation of $S^{n}$ which keep its base point $s_{n}$ fixed and interchanges the two hemispheres of $S^{n}$. This implies that for $n>1$ the group $\pi_{n}\left(X, x_{0}\right)$ is Abelian.

Definition 1.5. The infinite-dimensional sphere $S^{\infty}$ is a subspace of $\mathbb{R}^{\infty}$ consisting of all real sequences $\left(x_{1}, x_{2}, x_{3}, \cdots\right)$ such that $x_{1}{ }^{2}+x_{2}{ }^{2}+x_{3}{ }^{2}+\cdots=1$.

Definition 1.6. If $x$ is a point of $S^{n}$, then its antipode is a point $-x \in S^{n}$. A continuous map $f: S^{n} \rightarrow S^{m}$ is said to be antipode preserving if for every $x \in S^{n}$, $f(-x)=-f(x)$. 
Lemma 1.1. For every continuous map $f: S^{2} \rightarrow \mathbb{R}^{2}$ there exists a pair of antipodal points $x$ and $-x$ in $S^{2}$ such that $f(-x)=f(x)$.

Proof. If possible let $f(-x) \neq f(x)$, for every $x \in S^{2}$. Define $g: S^{2} \rightarrow S^{1}$ by

$$
x \mapsto \frac{f(x)-f(-x)}{\|f(x)-f(-x)\|} .
$$

This implies that $g$ is a continuous map such that $g(-x)=-g(x)$, for all $x \in S^{2}$. This is a contradiction because there is no continuous map $g: S^{2} \rightarrow S^{1}$ such that $g(-x)=-g(x)$.

In Section 2, we construct some results on finite and infinite symmetric product of spheres and we construct and compute the homotopy group of symmetric product of spheres.

\section{Some Results on Finite AND Infinite SYMMETRIC PRODUCT OF $S^{n}$ AND $S^{\infty}$}

Dold and Tom, [5], showed that $S P^{\infty}\left(S^{n}\right)=K(\mathbb{Z}, n)$, which is an EilenbergMacLane space with homotopy group $\pi_{k}(K(\mathbb{Z}, n))$ are all zero except for $k=n$.

Theorem 2.1. The natural inclusion $S^{n} \hookrightarrow S P^{\infty}\left(S^{n}\right)$ of the circle in its infinite symmetric product is a homotopy equivalence. Therefore

$$
\pi_{k}\left(S P^{\infty}\left(S^{n}\right)\right) \cong \pi_{k}\left(S^{n}\right)=\left\{\begin{array}{ccc}
\mathbb{Z} & \text { for } & k=n \\
0 & \text { for } & k \neq n
\end{array}\right.
$$

Proof. Since $S P^{\infty}\left(S^{n}\right)$ is the Eilenberg-MacLane space $\mathrm{K}(\mathbb{Z}, n)$, the theorem follows.

Theorem 2.2. $\pi_{k}\left(S P^{\infty}\left(\Sigma S^{n}\right)\right)=\left\{\begin{array}{ccc}\mathbb{Z} & \text { for } & k=n \\ 0 & \text { for } & k \neq n\end{array}\right.$

Proof. Since $\pi_{k}\left(S P^{\infty}\left(\Sigma S^{n}\right)\right) \cong \pi_{k-1}\left(S P^{\infty}\left(S^{n-1}\right)\right)$ and using the Theorem 2.1 the theorem follows.

Proposition 2.1. The infinite-dimensional sphere $S^{\infty}$ is contractible.

Proof. Consider the map $F: S^{\infty} \times I \rightarrow S^{\infty}$ defined by

$$
\left(x_{1}, x_{2}, \cdots, t\right) \mapsto\left((1-t) x_{1}, t x_{1}+(1-t) x_{2}, \cdots\right) / N_{t},
$$

where $N_{t}=\left[\left((1-t) x_{1}\right)^{2}+\left(t x_{1}+(1-t) x_{2}\right)^{2}+\cdots\right]^{\frac{1}{2}}$, which is norm of the nonzero vector of the numerator. We can write $F_{t}\left(x_{1}, x_{2}, \cdots\right)=F\left(x_{1}, x_{2}, \cdots, t\right)$. Then $F_{0}\left(x_{1}, x_{2}, \cdots\right)=\left(x_{1}, x_{2}, \cdots\right)$, since $N_{0}=1$ and $F_{1}\left(x_{1}, x_{2}, \cdots\right)=\left(0, x_{1}, x_{2}, \cdots\right)$, since $N_{1}=1$. Then $F_{0}$ is the identity map $I_{d}: S^{\infty} \rightarrow S^{\infty}$, the image of $F_{1}$ is the set $X=\left\{x \in S^{\infty}: x_{1}=0\right.$ and $\left.F: F_{0} \simeq F_{1}\right\}$.

Consider another homotopy $H: X \times I \rightarrow S^{\infty}$ defined by

$$
H\left(x_{1}=0, x_{2}, x_{3}, \cdots, t\right) \mapsto\left(t,(1-t) x_{2},(1-t) x_{3}, \cdots\right) / N_{t}^{\prime},
$$

where $N_{t}^{\prime}=\left[t^{2}+\left((1-t) x_{2}\right)^{2},\left((1-t) x_{3}\right)^{2}+\cdots\right]^{\frac{1}{2}}$. If $i: X \hookrightarrow S^{\infty}$ is the inclusion map, then $H: i \simeq c$, where $c$ is a constant map. 
Let $H \star F: S^{\infty} \times I \rightarrow S^{\infty}$ be defined by $(H \star F)(t)=F(x, 2 t)$ for $0 \leq t \leq 1 / 2$ and $H(x, 2 t-1)$ for $1 / 2 \leq t \leq 1$, where $x=\left(x_{1}, x_{2}, x_{3}, \cdots\right) \in S^{\infty}$.

Thus $H \star F$ is a contraction and hence $S^{\infty}$ is a contractible space.

Proposition 2.2. The infinite symmetric product space of $S^{\infty}$, i.e. $S P^{\infty}\left(S^{\infty}\right)$ is contractible, where $S^{\infty}$ is infinite-dimensional sphere.

Proof. Using Definition 1.1. Definition 1.5 and Proposition 2.1, it follows.

Proposition 2.3. $\pi_{k}\left(S P^{\infty}\left(S^{\infty}\right)\right) \cong \pi_{k}\left(S^{\infty}\right)=0$, for all $k$.

Proof. From the Theorem 2.1 and Proposition 2.2, it follows.

Proposition 2.4. Given a positive integer $n, \pi_{k}\left(S^{n}\right) \cong \begin{cases}\mathbb{Z} & \text { for } k=n \\ 0, & \text { otherwise }\end{cases}$

Proof. Let $S_{1}=\left\{x \in S^{n}: x_{n}>\frac{-1}{2}\right\}$ and $S_{2}=\left\{x \in S^{n}: x_{n}<\frac{1}{2}\right\}$. Since $S_{1}$ and $S_{2}$ are contractible spaces, using Mayer-Vietoris sequence and [9], it follows.

Let $\mathbf{C}$ be the category whose objects are topological spaces having the same homotopy type of finite $\mathrm{CW}$-complexes and morphisms are maps of such spaces and let $S P^{\infty}$ be a covariant functor from the category of pointed topological spaces and base point preserving continuous maps to the category of pointed topological spaces and base point preserving continuous maps, the we have the following:

Proposition 2.5. Let $S P^{\infty}$ be a covariant functor and $\Sigma$ is a suspension functor. Then $\pi_{k}\left(S P^{\infty}\left(\Sigma S^{n}\right)\right) \cong \pi_{k-1}\left(S^{n-1}\right)$.

Proof. Since $\pi_{k}\left(S P^{\infty}\left(\Sigma S^{n}\right)\right) \cong \pi_{k-1}\left(S P^{\infty}\left(S^{n-1}\right)\right)$ and using [7] and [8], it follows.

Finally we have the following theorem.

Theorem 2.3. $\pi_{k}\left(S P^{\infty}\left(S^{n}\right)\right)=\left\{\begin{array}{ccc}\mathbb{Z} & \text { for } & k=n \\ 0 & \text { for } & k \neq n\end{array}\right.$

Proof. It follows from the Proposition 2.4, Proposition 2.5 and the Theorem 2.2

\section{Some APPlications of Homotopy of INFINITE SYMMETRIC PRODUCTS OF SPHERES}

Proposition 3.1. $\pi_{k}\left(S P^{\infty}\left(S^{m}\right)\right) \nRightarrow \pi_{k}\left(S P^{\infty}\left(S^{n}\right)\right)$.

Proof. Let us assume that $0 \leq m<n$. Then using Theorem 2.3, we have $\pi_{n}\left(S P^{\infty}\left(S^{m}\right)\right)=0$ and $\pi_{n}\left(S P^{\infty}\left(S^{n}\right)\right)=\mathbb{Z}$. Thus, $\pi_{k}\left(S P^{\infty}\left(S^{m}\right)\right) \varsubsetneqq \pi_{k}\left(S P^{\infty}\left(S^{n}\right)\right)$.

Proposition 3.2. The spheres $S^{m}$ and $S^{n}$ are not homotopically equivalent, where $m$ and $n$ are two distinct non-negative integers.

Proof. Using Proposition 3.1 it follows. 
Proposition 3.3. $\pi_{k}\left(S P^{\infty}\left(S^{m}\right)\right) \nsim \pi_{k}\left(S P^{\infty}\left(S^{n}\right)\right)$

Proof. Using the Proposition 3.1 and Proposition 3.2 , it follows.

Proposition 3.4. If $m \neq n$, then the spheres $S^{n}$ and $S^{m}$ are not homeomorphic.

Proof. Using Proposition 3.2 it follows.

Proposition 3.5. The Euclidean space $\mathbb{R}^{m}$ and $\mathbb{R}^{n}$ are not homeomorphic, where $m \neq n$.

Proof. Using Proposition 3.2 and Proposition 3.4 , it follows.

Proposition 3.6. Let $m, n$ be integers such that $m>n \geq 0$. Then there does not exist a continuous map $f: S^{m} \rightarrow S^{n}$ preserving the antipodal points.

Proof. If possible, let there exist a continuous map $f: S^{m} \rightarrow S^{n}$ such that $f(x)=$ $f(-x)$, for all $x \in S^{m}$. Let $S^{n}$ is obtained from $S^{m}$ by giving the last $m-n$ coordinates equal to zero and $i: S^{n} \hookrightarrow S^{m}$ is the usual inclusion map. Then the composition $i \circ f: S^{m} \rightarrow S^{m}$ is antipodal preserving continuous map and so $\operatorname{deg}(i \circ f)$ must be odd integer. Again, $(i \circ f)_{*}=i_{*} \circ f_{*}$. The composition of $f_{*}: \pi_{m}\left(S P^{\infty}\left(S^{m}\right)\right) \rightarrow \pi_{m}\left(S P^{\infty}\left(S^{n}\right)\right)$ and $i_{*}: \pi_{m}\left(S P^{\infty}\left(S^{n}\right)\right) \rightarrow \pi_{m}\left(S P^{\infty}\left(S^{m}\right)\right)$ is the trivial homomorphism, by Theorem 2.3. Thus we have a contradiction.

\section{ACKNOWLEDGMENT}

The authors are grateful to the anonymous referee for his/her valuable suggestions which considerably improved the presentation of the paper. The first and third author are very thankful to THE GOVERNMENT OF WEST BENGAL, DEPARTMENT OF SCIENCE AND TECHNOLOGY AND BIOTECHNOLOGY that encouraged them to research this topic with sufficient grant. The second author is thankful to the Council of Scientific and Industrial Research, HRDG, India, for granting him Junior Research Fellowship (File No. 08/525(0006)/2019-EMR-I) during the tenure of which this work was done.

\section{REFERENCES}

[1] A. Adhikari, P. K. Rana, A study of functors associated with Topological groups, Studia Univ. "Babes-Bolyai", Mathematica, Vol XLVI, No. 4, Dec. 2001.

[2] M. R. Adhikari, Construction of general homology theory, Cal. Math. Society 71(6) (1979), 396-398.

[3] M. R. Adhikari, Basic Algebraic Topology and its Applications, Springer, 2016.

[4] A. Dold, Homology of Symmetric Products and Other Functors of Complexes, Vol.68, No.1 (Jul.1958), 54-80.

[5] A. Dold, R. Thom, Quasifibres and Symmetric Products, Ann. of Math. 67 (1958), 239-281.

[6] A. Hatcher, Algebraic Topology, Cambridge University Press, 2002.

[7] P. K. Rana, A study of the group of covering transformation through functors, Mat. Bilten 33(LIX) (2009), 21-24.

[8] E. H. Spanier, Infinite symmetric product of functors spaces and duality, Ann. of Math. 69 (1959), 142-198. 
[9] E. H. Spanier, Algebraic Topology, Mc Graw-Hill, New York.

[10] P. K. Rana, A Study of some functors and their ralations, The Journal of Indian Academy of Mathematics, Vol.34(1) (2012), 73-81.

1 Department Of Mathematics,

Ramakrishna Mission Vivekananda Centenary College,

PO-Rahara,Kolkata-700118, West Bengal, India

Email address: pkranabgc@gmail.com

Email address: 99bmandal@gmail.com

2 JRF, Project under WBSTBD

Email address: sarmad786hossain@gmail.com

Received 30.4.2021

Revised 31.7.2021

Accepted 11.8.2021 